\title{
ALMOST KRULL RINGS
}

\author{
E. JESPERS and P. WAUTERS
}

(Received 12 December 1984; revised 24 September 1985)

Communicated by R. Lidl

\begin{abstract}
The notion of an almost Krull domain is extended to rings satisfying a polynomial identity. Some general structural results are obtained. We also prove that skew polynomial rings $R[X, \sigma]$ remain almost Krull if $R$ is an almost Krull ring. Finally, we study when semigroup rings $R[S]$ are almost Krull rings, in the case when the group of quotients of $S$ has the ascending chain condition on cyclic subgroups. An example is included to show that the general (semi-) group ring case is much more difficult to deal with.
\end{abstract}

1980 Mathematics subject classification (Amer. Math. Soc.): 16 A 15, 16 A 18, 16 A 38.

\section{Introduction}

In the literature (cf. [9] and [11]) there appears the notion of an almost Dedekind domain and an almost Krull domain. An almost Dedekind (respectively almost Krull) domain $R$ is defined as a commutative integral domain such that $R_{P}$ is a Dedekind domain (respectively Krull domain) for all non-zero prime ideals $P$ of $R$. In particular, an almost Krull domain is almost Dedekind if and only if all non-zero prime ideals are maximal. Moreover, the notion of an almost Krull domain is closed under polynomial extensions. In this paper we extend this to rings satisfying a polynomial identity. We obtain some general structural results

The second named author is a senior research assistant at the National Fund for Scientific Research (Belgium).

This research was done while the second named author visited the Department of Mathematics of the University of Stellenbosch, which he thanks for its hospitality. He was also supported by an N.F.W.O. grant (Belgium).

(C) 1986 Australian Mathematical Society $0263-6115 / 86$ \$A2.00 +0.00 
about these rings. We also prove that if $R$ is an almost Krull ring, and if $R[X, \sigma]$ is a skew polynomial ring which is a prime P.I. ring, then $R[X, \sigma]$ is an almost Krull ring. Finally, we offer an example of a (commutative) group ring which is an almost Krull domain but not a Krull domain.

\section{Definition and properties}

Throughout, all rings are associative with unity. Ideal will always mean two-sided ideal. If $R$ is a ring satisfying a polynomial identity, then we say, for brevity, that $R$ is a P.I. ring. We refer to [16] for results on P.I. rings. Spec $R$ denotes the set of prime ideals of $R$. We refer to [13] for the definition of a maximal order.

Definition 1.1. Let $R$ be a prime P.I. ring with classical ring of quotients $Q$. Then $R$ is said to be an almost Krull ring (respectively almost Asano-order) if for all $0 \neq p \in \operatorname{Spec} C$ (where $C=Z(R)$, the centre of $R$ ), $R_{p}=\{q \in Q \mid c q \in R$ for some $c \in C \backslash p\}$ is a maximal order with centre a Krull domain (respectively a Dedekind domain).

In particular, if $R$ is a Krull ring, i.e. $R$ is a maximal order with centre a Krull domain, then it follows from [4] that $R$ is an almost Krull ring. Similarly, if $R$ is an Asano-order, hence bounded and therefore hereditary (we refer to [4] for definitions and proofs), then $R$ is an almost Asano-order by [15].

\section{LemMA 1.2. Let $R$ be an almost Krull ring. Then $R$ is a maximal order.}

Proof. Let $I$ be a non-zero ideal of $R$, and let $\alpha \in Q$ be such that $\alpha I \subset I$. then $\alpha I R_{p} \subset I R_{p}$ for each maximal ideal $p$ of $C$. Since $I R_{p}$ is a two-sided ideal of $R_{p}$ and $R_{p}$ is a maximal order, it follows that $\alpha \in R_{p}$. Hence

$$
\alpha \in \bigcap_{p \max } R_{p}=R
$$

Let $R$ be an almost Krull ring. For all $0 \neq p \in \operatorname{Spec} C$ we have that $Z\left(R_{p}\right)=C_{p}$ is a Krull domain. Therefore $C$ is an almost Krull domain. In particular, $C$ is completely integrally closed. Hence, by [17], $R$ is integral over $C$. Moreover, since $C$ is also integrally closed, lying over (LO), going up (GU), incomparability (INC) and going down (GD) hold for prime ideals (cf. [1]). 
Let $R$ be an almost Krull ring. Then

$$
R=\bigcap_{0 \neq p \in \operatorname{Spec} C} R_{p} .
$$

But each $R_{p}$ is a maximal order over a Krull domain. So, by [4] (see also [13]),

$$
R_{p}=\bigcap_{p_{i}^{\prime} \in X^{1}\left(C_{p}\right)}\left(R_{p}\right)_{p_{i}^{\prime}}
$$

where $X^{1}(R)$ denotes the set of height one prime ideals of $R$. Moreover, each $\left(R_{p}\right)_{p_{i}^{\prime}}$ is a quasi-local Asano-order. (A ring $R$ is said to be quasi-local if $J(R)$, the Jacobson radical of $R$, is the unique maximal ideal of $R$ and $R / J(R)$ is Artinian.) Let $p_{i}^{\prime} \in X^{1}\left(C_{p}\right)$. Then $p_{i}^{\prime}=C_{p} p_{i}$, where $p_{i}=p_{i}^{\prime} \cap C$. By elementary properties of localisation, it follows that $p_{i} \in X^{1}(C)$. It is clear that

$$
\left(R_{p}\right)_{p_{i}^{\prime}}=R_{p_{j}}
$$

and therefore

$(* * *)$

$$
R_{p}=\bigcap_{\substack{p_{i} \in X^{1}(C) \\ p_{i} \subset p}} R_{p_{i}} .
$$

Combination of $(*)$ and $(* * *)$ gives $R=\bigcap_{p \in X^{1}(C)} R_{p}$. Note that if $p \in X^{1}(C)$, then $R_{p}$ is a quasi-local Asano-order by $(* *)$, and so $C_{p}$ is a discrete valuation ring.

We remark that in expression $(* * *)$, the set $\left.p_{i} \in X^{1}(C) \mid p_{i} \subset p\right\}$ is non-empty. For let $0 \neq p \in \operatorname{Spec} C$. Then $p_{p} \in \operatorname{Spec}\left(C_{p}\right)$. Since $C_{p}$ is a Krull domain, $p_{p} \supset q^{\prime}$ and $q^{\prime} \in X^{1}\left(C_{p}\right)$. But then $q=\left(q^{\prime} \cap C\right) \in X^{1}(C)$ and $q \subset p$.

It also follows from this observation, and from (LO), (INC) and (GD), that if $R$ is an almost Krull ring and $0 \neq P \in \operatorname{Spec} R$, then $P \supset P^{\prime}$ for some $P^{\prime} \in X^{1}(R)$.

If $P \in X^{1}(R)$, then $p=P \cap C \in X^{1}(C)$ by (GD). Conversely, if $p \in X^{1}(C)$, then, by (INC), there exists $P \in X^{1}(R)$ such that $p=P \cap C$. So the map $X^{1}(R) \rightarrow X^{1}(C): P \rightarrow(P \cap C)$ is surjective. We show now that it is also injective. Let $p \in X^{1}(C)$. Then $R_{p}$ is a quasi-local Asano-order. Each ideal of $R_{p}$ is of the form $A_{p}=\left\{c^{-1} a \mid c \in C \backslash p, a \in A\right\}$, where $A$ is an ideal of $R$ such that $(A \cap C) \subset p$. In particular, if $P \in X^{1}(R)$ is such that $P \cap C=p$, then $P_{p}$ is an ideal of $R$. Moreover, it is easy to check that $P_{p} \cap R=P$, and then it follows that $P_{p}$ is a non-zero prime ideal of $R_{p}$. But $R_{p}$ has only one non-zero prime ideal, namely $J\left(R_{p}\right)$. So $P=J\left(R_{p}\right) \cap R$, i.e. $P$ is completely determined by $p$, and so $P$ is unique.

Let $P \in X^{1}(R)$ and let $p=P \cap C \in X^{1}(C)$. Let $C(P)$ (respectively $C\left(P_{p}\right)$ ) denote the elements of $R$ (respectively $R_{p}$ ) which are regular modulo $P$ (respectively $\left.P_{p}\right)$. It is clear that $C(P) \subset C\left(P_{p}\right)$. Let $x \in C\left(P_{p}\right)$. Then $\bar{x}=x+P_{p}$ is regular in $R_{p} / P_{p}$, which is simple Artinian. Hence $\bar{x}$ is invertible, i.e. $a=1-x y$ $\in P_{p}$ for some $y \in R_{p}$. But $P_{p}=J\left(R_{p}\right)$, so $x y=1+a$ is invertible, and so $x$ is 
invertible. Therefore, if $x \in C(P)$, then $R_{p} x=x R_{p}=R_{p}$. We claim that $C(R)$ is a left and right Ore-set in $R$. For let $x \in C(P)$ and $a \in R$. Then $R_{p} x=R_{p}$, so $a=\alpha x$ for some $\alpha \in R_{p}$. But $\beta=c \alpha \in R$ for some $c \in C \backslash p$, and so $c a=\beta x \in C(P) a \cap R x$. Similarly, $C(P)$ is a right Ore-set, and it is clear then that $R_{C(P)}=R_{p}$, where $R_{C(P)}=\left\{x^{-1} r \mid x \in C(P), r \in R\right\}$. This establishes

Lemma 1.3. Let $R$ be an almost Krull ring, let $P \in X^{1}(R)$ and let $p=(P \cap C)$. Then $C(P)$ is an Ore-set and $R_{C(P)}=R_{p}$.

LEMMA 1.4. Let $R$ be an almost Krull ring and $S$ a multiplicatively closed subset of $C$, where $C$ is the centre of $R$. Then $R_{S}$ is again an almost Krull ring, where $R_{S}=\left\{c^{-1} r \mid c \in S, r \in R\right\}$.

Proof. Each non-zero prime ideal of $Z\left(R_{S}\right)=C_{S}$ is of the form $p_{S}$, where $p$ is a non-zero prime ideal of $C$ such that $p \cap S=\varnothing$. But obviously $\left(R_{S}\right)_{p_{s}}=R_{p}$, which is a Krull ring. Hence $R_{S}$ is an almost Krull ring.

Proposition 1.5. Let $R$ be an almost Krull ring. Then the following conditions are equivalent:

(1) $R$ is an almost Asano-order;

(2) $C$ is an almost Dedekind domain;

(3) all non-zero prime ideals of $C$ are maximal;

(4) all non-zero prime ideals of $R$ are maximal.

Proof. $(1) \Leftrightarrow(2)$ : this is clear from the definition of almost Asano-order and from (INC).

(2) $\Rightarrow(3)$ : let $0 \neq p \in \operatorname{Spec} C$. Then $C_{p}$ is a local Dedekind domain, hence a discrete valuation ring. Therefore $p$ is a minimal non-zero prime ideal.

(3) $\Rightarrow(2)$ : let $0 \neq p \in \operatorname{Spec} C$. Then $C_{p}$ is a Krull domain. But all non-zero prime ideals of $C_{p}$ are maximal since the same holds for $C$. So $C_{p}$ is a Dedekind domain.

(3) $\Rightarrow(4)$ : this follows from (INC).

(4) $\Rightarrow(3)$ : this follows from (GU).

Proposition 1.6. Let $R$ be an almost Krull ring. Then the following conditions are equivalent:

(1) $R$ is a Krull ring;

(2) for each $r \in R$ which is regular, $r \notin C(P)$ for only finitely many $P \in X^{1}(R)$;

(3) for all $0 \neq c \in C, c \in p$ for only finitely many $p \in X^{1}(C)$;

(4) $C$ is a Krull domain. 
Proof. (1) $\Rightarrow$ (2): this is clear by Proposition 1.8 of [5].

(2) $\Rightarrow$ (3): if $P \in X^{1}(R)$, then $p=(P \cap C) \in X^{1}(C)$ and $(C \backslash p)=C(P) \cap$ $C$. So if $0 \neq c \in C$, then $c \notin p$ for almost all $p \in X^{1}(C)$.

(3) $\Rightarrow$ (4): since $C=\bigcap_{p \in X^{1}(C)} C_{p}$, the result follows from Proposition 1.9 of [8].

(4) $\Rightarrow$ (1): this is clear because $R$ is a maximal order.

We now obtain some structural results about almost Krull rings that form finite modules over their centres. We first need the following lemma.

LEMMA 1.7. Let $R$ be a prime P.I. ring which is a finite module over its centre $C$. If $R$ is a maximal order and $C$ an almost Krull domain, then $R_{p}$ is a maximal order for every $p \in X^{1}(C)$.

Proof. Let $p \in X^{1}(C)$. Since $C$ is an almost Krull domain, $C_{p}$ is a discrete valuation ring, and in particular a noetherian ring. By a result of Formanek (cf. e.g. [16]), it follows that $R_{p}$, too, is noetherian.

To prove that $R_{p}$ is a maximal order it suffices to show that $\left(I:_{l} I\right) \subset R$ (and $\left.\left(I:{ }_{r} I\right) \subset R\right)$ for every non-zero ideal $I$ of $R_{p}$. Let $I$ be such an ideal. Since $R_{p}$ is noetherian, $I=\sum_{j=1}^{k} R_{p} y_{j}$ for some elements $y_{j} \in I \cap R, 1 \leqslant j \leqslant k$. Let $A=$ $\sum_{j=1}^{k} R y_{j}$. Then $A R$ is a non-zero ideal of $R$. Because of the assumption, $R=\sum_{i=1}^{m} C r_{i}$ for certain $r_{i} \in R, 1 \leqslant i \leqslant m$. Suppose $\alpha \in(I:, I)$. Then $\alpha r_{i} y_{j} \in I$ for $1 \leqslant i \leqslant m, 1 \leqslant j \leqslant k$. Hence there exists a $c \in C \backslash p$ such that $c \alpha r_{i} y_{j} C \subset A$ for all $i, j$. Hence, $c \alpha R y_{j}=c \alpha\left(\sum_{i=1}^{m} r_{i} C\right) y_{j} \subset A$ for all $1 \leqslant j \leqslant k$. Therefore, $c \alpha A=c \alpha \sum_{j=1}^{k} R y_{j} \subset A$, and thus $c \alpha A R \subset A R$. Because $R$ is a maximal order, it follows that $c \alpha \in R$, and in particular that $\alpha \in R_{p}$. So we have shown that $(I:, I) \subset R$. Similarly, $(I:, I) \subset R$.

Proposition 1.8. Let $R$ be a prime P.I. ring which is a finite module over its centre $C$. If $C$ is an almost Krull domain, and if $R$ is a maximal order such that for all $0 \neq p \in \operatorname{Spec} C, R_{p}$ is a projective $C_{p}$-module, then $R$ is an almost Krull ring.

Proof. Because $C$ is an almost Krull domain, we only have to prove that $R_{p}$ is a maximal order for any $0 \neq p \in \operatorname{Spec} C$. By Lemma 1.7 and Proposition 1.3 in [7] it remains to prove that $\operatorname{Hom}_{C_{p}}\left(\operatorname{Hom}_{C_{p}}\left(R_{p}, C_{p}\right), C_{p}\right)$, (i.e. the bidual of $R_{p}$ ) is naturally isomorphic to $R_{p}$. Since $C_{p}$ is a local domain, and since, by assumption, $R_{p}$ is a finitely generated free $C_{p}$-module, the above statement is well-known.

It is now known to the authors whether one can drop the assumption of projectiveness in the above proposition. But for almost Asano-orders we do obtain a nice characterisation. 
COROLLARY 1.9. Let $R$ be a prime P.I. ring which is a finite module over its centre $C$. Then the following are equivalent:

(1) $R$ is an almost Asano-order;

(2) $R$ is a maximal order and $C$ is an almost Dedekind domain.

Proof. (1) $\Rightarrow$ (2): this follows from Lemma 1.2 and Proposition 1.5 .

(2) $\Rightarrow(1)$ : because of Proposition 1.5, it is sufficient to show that $R$ is an almost Krull ring. Now, for each $0 \neq p \in \operatorname{Spec} C . R_{p}$ is an finitely generated torsion free $C_{p}$-module, and hence a projective $C_{p}$-module, because $C_{p}$ is a Dedekind domain (cf. [2]). Therefore the result follows from Proposition 1.8.

\section{Skew polynomial rings}

Let $R$ be a prime P.I. ring with classical ring of quotients $Q(R)$. If $\sigma \in \operatorname{Aut}(R)$, then clearly $\sigma$ extends in a unique way to an automorphism of $Q(R)$. Now G. Cauchon [3] has proved that if $A$ is a simple P.I. ring, then the skew polynomial ring $A[X, \sigma]$ is a prime P.I. ring if and only if $\sigma$ is power-inner, i.e. there exists $n>0$ and an invertible element $u$ of $A$ such that $\sigma^{n}(a)=u a u^{-1}$ for all $a \in A$. In particular $\sigma^{n}$ is the identity on $Z(A)$.

THEOREM 2.1. Let $R$ be a prime ring and let $\sigma \in$ Aut $R$ be such that $R[X, \sigma]$ is a prime P.I. ring. Suppose that $R$ is an almost Krull ring. Then $R[X, \sigma]$ is an almost Krull ring.

Proof. Let $Q(R)$ denote the classical ring of quotients of $R$. If $R[X, \sigma]$ is a prime P.I. ring, so is $Q(R)[X, \sigma]$. Hence, by the foregoing, $\sigma^{n}$ is the identity on $Z(Q(R))$ for some $n>0$. So if $c \in C$ (note that $C$ denotes the centre of $R$ ), then $d=c \sigma(c) \cdots \sigma^{n-1}(c) \in C$ and $\sigma(d)=d$, so $d \in C^{\sigma}=\{c \in C \mid \sigma(c)=c\}$. It follows that $Q(R)[X, \sigma]=\left(C^{\sigma}\right)^{-1} R[X, \sigma]$ and $Z(Q(R)[X, \sigma])=$ $\left(C^{\sigma}\right)^{-1} Z(R[X, \sigma])$. Also $Z(R[X, \sigma]) \cap R=C^{\sigma}$, as is easily seen.

We have to show that $R[X, \sigma]_{p}$ is a maximal order over a Krull domain for each $0 \neq p \in \operatorname{Spec}(Z(R[X, \sigma])$. So let $0 \neq p \in \operatorname{Spec}(Z(R[X, \sigma]))$. Suppose first that $p \cap C^{\sigma}=0$. Then $p Z(Q(R)[X, \sigma]) \cap Z(R[X, \sigma])=p$, and $p Z(Q(R)[X, \sigma])$ is a non-zero prime ideal of $Z(Q(R)[X, \sigma])$. A straightforward computation shows then that $R[X, \sigma]_{p}=Q(R)[X, \sigma]_{p Z(Q[X, \sigma])}$. But $Q[X, \sigma]$ is an Asano-order, since each two-sided ideal is principal (cf., for example, [3]). So $R[X, \sigma]_{p}=$

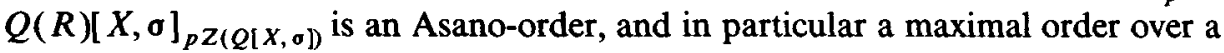
Krull domain.

Suppose now that $q=p \cap C^{\sigma} \neq 0$. Then $q \in \operatorname{Spec} C^{\sigma}$. Now $\sigma$ is an automorphism of $C$ of finite order. By a classical theorem of E. Noether, $C$ is 
integral over $C^{\boldsymbol{\sigma}}$. In particular, there exists a $Q \in \operatorname{Spec} C$ such that $Q \cap C^{\sigma}=q$. Moreover, by [2, Chapter V. 2.2. Theorem 2], if $P \in \operatorname{Spec} C$ is such that $P \cap C^{\sigma}=q$, then $P=\sigma^{i}(Q)$ for some $i>0$. Hence there are only finitely many prime ideals of $C$ lying over $q$. Now let $Q \in \operatorname{Spec} C^{\sigma}$ be such that $Q \cap C^{\sigma}=q$. We introduce some notation: let $S=C^{\sigma} \backslash q$ and $T_{i}=C \backslash \sigma^{i}(Q)$ for $0 \leqslant i \leqslant n-$ 1.

Claim 1. $R_{S}=\bigcap_{0 \leqslant i \leqslant n-1} R_{T_{i}}$.

Proof of Claim 1. If $c \in S$, then clearly, since $\sigma(c)=c, c \notin \sigma^{i}(Q)$ for all $i$, $0 \leqslant i \leqslant n-1$. So $c^{-1} \in \bigcap_{0 \leqslant i \leqslant n-1} R_{T_{i}}$. Conversely, let $\alpha \in \bigcap_{0 \leqslant i \leqslant n-1} R_{T_{i}}$. For each $i$, there exists $c_{i} \in T_{i}$ such that $c_{i} \alpha \in R$. Put $J=\sum_{i=0}^{n} c d_{i}$. Then $J$ is an ideal of $C, J \alpha \subset R$ and $J \not \subset \sigma^{i}(Q)$ for all $i$. But since $\sigma^{i}(Q) \in \operatorname{Spec} C$ for all $i$, we have $J \not \subset \bigcup_{i=0}^{n-1} \sigma^{i}(A)$. So there exists $c \in J$ such that $c \notin \sigma^{i}(Q)$ for all $i$, and hence such that $\sigma^{i}(c) \notin Q$ for all $i$. Put $b=c \sigma(c) \cdots \sigma^{n-1}(c)$. Then $b \in C^{\sigma}$, but $b \notin q$, for if $b \in q=Q \cap c^{\sigma}$, then $b=c^{\sigma}(c) \cdots \sigma^{n-1}(c) \in Q$, so that $\sigma^{i}(c) \in Q$ for some $i \geqslant 0$ (since $Q$ is prime), and this is a contradiction. Thus $b \in S$, and since $b \in J$, we have $b \alpha \in R$, and so $\alpha \in R_{S}$.

Claim 2. $R_{S}$ is a maximal order over a Krull domain.

Proof of Claim 2. Since $R$ is an almost Krull ring, and since $0 \neq \sigma^{i}(Q) \in$ Spec $C$, it follows that $R_{T_{i}}$ is a maximal order over a Krull domain. Since ideals of $R_{S}$ extend to ideals of $R_{T_{i}}$, it is immediate from Claim 1 that $R_{S}$ is a maximal order. Moreover, $Z\left(R_{S}\right)=\bigcap_{i} Z\left(R_{T_{i}}\right)$, which is a finite intersection of Krull domains, and so again a Krull domain.

Straightforward computations now show that $R[X, \sigma]_{S}=\left(R_{S}\right)[X, \sigma]$ and $R[X, \sigma]_{p}=\left(R[X, \sigma]_{S}\right)_{p_{S}}$. Combination of these facts gives that

$$
R[X, \sigma]_{p}=\left(R_{S}[X, \sigma]\right)_{p_{S}} \text {. }
$$

By Claim $2, R_{S}$ is a maximal order over a Krull domain, and hence a Krull ring in the sense of $M$. Charmarie [4]. Therefore, by [4], $R_{S}[X, \sigma]$ is a Krull ring, and also $\left(R_{S}[X, \sigma]\right)_{p_{S}}$ is a maximal order over a Krull domain, which, by $(*)$, is the desired result. This completes the proof.

\section{Semigroup rings}

In [6] a commutative Krull semigroup was defined. We say that a commutative cancellative semigroup $S$ is an almost Krull semigroup if and only if, for all 
$p \in \operatorname{Spec} S, S_{p}=\left\{c^{-1} s \mid c \in S \backslash p, s \in S\right\}$ is a Krull semigroup. Note that $p \in$ Spec $S$, i.e. $p$ is a prime ideal of $S$, if $S \backslash p$ is a subsemigroup of $S$.

Let $k$ be a field and $G$ a torsion free abelian group. Recall that $k[G]$ is a Krull domain if and only if $k[G]$ is a unique factorisation domain if and only if $G$ satisfies the ACC on cyclic subgroups (cf. [10], [12]).

LeMma 3.1. Let $R$ be a prime P.I. ring and $G$ a torsion free abelian group. If $R[G]$ is an almost Krull ring, then $R$ is an almost Krull ring. The converse holds if $G$ satisfies the ACC on cyclic subgroups.

Proof. Suppose that $R[G]$ is an almost Krull ring. Let $0 \neq p \in \operatorname{Spec} C$, where $C$ is the centre of $R$. Then $R[G]_{C \backslash p}=R_{p}[S]$ is again an almost Krull ring by Lemma 1.4, and so $R_{p}[S]$ is a maximal order by Lemma 1.2. Then it follows from [18] that $R_{p}$ is a maximal order for each $p \in \operatorname{Spec} C$. So to prove that $R$ is an almost Krull ring, it suffices to show that $C_{p}$ is a Krull domain for each $0 \neq p \in \operatorname{Spec} C$. Let $0 \neq p \in \operatorname{Spec} C$. Then, since $C[S]$ is an almost Krull domain, we have that $C[S]_{p[S]}$ is a Krull domain. But $k=Q(C)$ is a subfield of $Q(C[S])$. Because $C_{p}=C[S]_{p[S]} \cap k$, it follows that $C_{p}$ is a Krull domain.

Conversely, suppose that $R$ is an almost Krull ring and that $G$ satisfies the ACC on cyclic subgroups. Let $0 \neq p \in \operatorname{Spec} C[G]$. If $p \cap C=0$, then $p=p k[G]$ $\cap C[G]$, where $k=Q(C)$ and $0 \neq p k[G] \in \operatorname{Spec} k[G]$. But $R[G]_{p}=$ $Q(R)[G]_{p k[G]}$. Since $Q(R)$ is a simple P.I. ring and $G$ satisfies the ACC on cyclic subgroups, it follows that $Q(R)[G]$ is a maximal order over a Krull domain [18], and hence the same holds for $Q(R)[G]_{p k[G]}$ by [5]. If $0 \neq q=p \cap C$, then $R[G]_{p}=R_{q}[G]_{p_{q}}$, where $p_{q}=\left\{c^{-1} a \mid c \in C \backslash q, a \in p\right\}$. Now $R_{q}$ is a Krull ring, since $R$ is an almost Krull ring and since $0 \neq q \in \operatorname{Spec} C$. Because $G$ has the ACC on cyclic subgroups, it follows for the same reason as before that $R[G]_{p}$ is a maximal order over a Krull domain.

Proposition 3.2. let $R$ be a prime P.I. ring and $S$ a torsion free commutative cancellative semigroup such that the quotient group $Q(S)$ of $S$ satisfies the ACC on cyclic subgroups. Then the following are equivalent:

(1) $R[S]$ is an almost Krull ring;

(2) $S$ is an almost Krull semigroup, and $R$ is an almost Krull ring.

Proof. (1) $\Rightarrow(2)$ : since $R[G]=S^{-1} R[S]$, it is clear from Lemma 1.4 and Lemma 3.1 that $R[G]$ and $R$ are almost Krull rings. Let $q \in \operatorname{Spec} S$. Then $0 \neq C[q] \in \operatorname{Spec} C[S]$, and so $C[S]_{C[q]}$ is a Krull domain. But $G=Q(S)$ is a subgroup of $Q(C[S]) \backslash\{0\}$. Since $C[S]_{C[q]} \cap G=S_{q}$, and since $C[S]_{C[q]} \backslash\{0\}$ is a Krull semigroup (cf. [19]), it follows that $S_{q}$ is a Krull semigroup. 
(2) $\Rightarrow$ (1): let $0 \neq p \in \operatorname{Spec} C[S]$. If $p \cap S=\varnothing$, then $R[S]_{p}=R[G]_{p C[G]}$, $p C[G] \in \operatorname{Spec} C[G]$ and $p=p C[G] \cap C[S]$. By Lemma $3.1 R[G]$ is an almost Krull ring; hence it follows that $R[S]_{p}=R[G]_{p C[G]}$ is a Krull ring. If $q=p \cap S$ $\neq \varnothing$, then $q \in \operatorname{Spec} S$, so that $R[S]_{p}=R\left[S_{q}\right]_{p_{q}}$, where $p_{q}=\left\{c^{-1} a \mid c \in S \backslash q\right.$, $a \in p\}$. If $p_{q} \cap C=0$, then

$$
R[S]_{p}=R\left[S_{q}\right]_{p_{q}}=Q(R)\left[S_{q}\right]_{p_{q} k\left[S_{q}\right]} .
$$

Since $S_{q}$ is a Krull semigroup, and since $G$ satisfies the ACC on cyclic subgroups, it follows that $Q(R)\left[S_{q}\right]$ is a Krull ring (cf. [18]), and, as before, the same holds for $R[S]_{p}$. If $m=p_{q} \cap C \neq 0$, then

$$
R[S]_{p}=R\left[S_{q}\right]_{p_{q}}=R_{m}\left[S_{q}\right]_{\left(p_{q}\right)_{m}} .
$$

Since $R_{m}$ is a Krull ring (by Lemma 1.3), and since $S_{q}$ is a Krull semigroup, the result follows by the same argument as before.

COROLlary 3.3. The polynomial ring $R\left[\left(X_{i}\right)_{i \in I}\right]$ (I arbitrary) is an almost Krull ring if and only if $R$ is an almost Krull ring.

Proof. Let $S$ be the free commutative semigroup generated by $\left\{X_{i} \mid i \in I\right\}$. Obviously $R\left[\left(X_{i}\right)_{i \in I}\right]=R[S]$. Moreover, it is clear from [6] that $S$ is a Krull semigroup and that $Q(S)$ has the ACC on cyclic subgroups. The result follows now from Lemma 3.1 and Proposition 3.2.

Let $C$ be a commutative integral domain. It is shown in [19] that $C$ is a Krull domain if and only if $C \backslash\{0\}$ is a Krull semigroup. Therefore $C \backslash\{0\}$ is an almost Krull semigroup if and only if $C$ is an almost Krull domain. Since there exist almost Krull domains which are not Krull domains, there also exist almost Krull semigroups which are not Krull semigroups. We give now an example of a group ring $k[G]$ which is an almost Krull domain but not a Krull domain.

EXAMPLE 3.4. Let $p$ be a prime number, and let

$$
G=\operatorname{grp}\left\langle x, x^{1 / p}, x^{1 / p^{2}}, \ldots, x^{1 / p^{n}}, \ldots\right\rangle .
$$

Then $G$ is a torsion free commutative group which does not satisfy the ACC on cyclic subgroups. So $k[G]$ is not a Krull domain for any field $k$. We show now that if $k$ is a field which is not of characteristic $p$, then $R=k[G]$ is an almost Dedekind domain. Clearly $G=\cup_{n=1}^{\infty} G_{n}$ where $G_{n}=\operatorname{grp}\left\langle x, \ldots, x^{1 / p^{n}}\right\rangle=$ $\operatorname{grp}\left\langle x^{1 / p^{n}}\right\rangle$, an infinite cyclic group. Let $R_{n}=k\left[G_{n}\right]$. Then $k\left[G_{n}\right] \cong k\left[T, T^{-1}\right]$ is obviously a Dedekind domain and $R=\bigcup_{n=1}^{\infty} R_{n}$. Let $0 \neq P \in \operatorname{Spec} R$. Then $P=\bigcup_{n=1}^{\infty} P_{n}$, where $P_{n}=P \cap R_{n} \in \operatorname{Spec} R_{n}$. Note that $R_{n+1} \cong R_{n}^{t}\left[C_{p}\right]$, a twisted group ring over the cyclic group of order $p$. In particular, $R_{n+1}$ is a finite 
$R_{n}$-module, so $R_{n+1}$ is integral over $R_{n}$. Hence, $P \neq 0$, so $0 \neq P \cap \operatorname{Spec} R_{n}$, but since $R_{n}$ is integral over $R_{n-1}$, we have $0 \neq\left(P \cap R_{n}\right) \cap R_{n-1}=P \cap R_{n-1} \in$ Spec $R_{n-1}$. We introduce some notation: let $V_{n}=\left(R_{n}\right)_{P_{n}}$ and $B_{n}=\left(P_{n}\right)_{P_{n}}$ for all $n \geqslant 1$. Clearly $R_{P}=\cup_{n=1}^{\infty} V_{n}$ and $V_{n} \subset V_{n+1}$ for all $n$. Since each $R_{n}$ is a Dedekind domain, $V_{n}$ is a discrete valuation ring for each $n$, with unique maximal ideal $B_{n}$. We claim that $V_{n+1} B_{n}=B_{n+1}$. Now $R_{n+1} / R_{n+1} P_{n} \cong\left(R_{n} / P_{n}\right)^{t}\left[C_{p}\right]$. But $R_{n} / P_{n}$ does not have characteristic $p$, and so by [14], $R_{n+1} / R_{n+1} P_{n}$ is a semiprime ring, i.e. $R_{n+1} P_{n}$ is a semiprime ideal of $R_{n+1}$. Since $R_{n+1}$ is a Dedekind domain, it follows that $R_{n+1} P_{n}=Q_{1} Q_{2} \cdots Q_{k}$, where each $Q_{i}$ is a maximal ideal of $R_{n+1}$, and where $Q_{i} \neq Q_{j}$ if $i \neq j$. Note that $P_{n+1}$ is one of the $Q_{i}$, say $P_{n+1}=Q_{1}$. But $V_{n+1} B_{n}=\left(R_{n+1} P_{n}\right)_{P_{n+1}}=\left(P_{n+1} Q_{2} \cdots Q_{k}\right)_{P_{n+1}}=B_{n+1}$, since if $Q_{i} \neq P_{n+1}$, then $\left(Q_{i}\right)_{P_{n+1}}=V_{n+1}$. The fact that $V_{n+1} B_{n}=B_{n+1}$ implies that the valuation $v_{n+1}$ on $V_{n+1}$ restricts to the valuation $v_{n}$ associated with $V_{n}$. Therefore

$$
v: Q\left(R_{p}\right) \rightarrow \mathbf{Z}: a \rightarrow v_{n}(a),
$$

where $a \in Q\left(V_{n}\right)$ is a well-defined valuation with associated discrete valuation ring $R_{p}$. Therefore $R$ is an almost Dedekind domain.

We note that if $k$ has characteristic $p$, then $R$ is not an almost Krull domain. Suppose that $R=k[G]$ is an almost Krull domain. Let $\omega k[G]$ be the augmentation ideal of $k[G]$. Then $\omega k[G]$ is a maximal ideal, and so $S=k[G]_{\omega k[G]}$ is a Krull domain. Now, for each $n>0$, we have

$$
1-x^{1 / p^{n}}=\left(1-x^{1 / p^{n+1}}\right) \alpha,
$$

where $\alpha=\left(1+x^{1 / p^{n+1}}+x^{2 / p^{n+1}}+\cdots+x^{(p-1) / p^{n+1}}\right)$. But since char $K=p$, we have $\alpha \in \omega[G]$, so $\alpha$ is not a unit in $S=k[G]_{\omega k[G]}$. Therefore, we have

$$
S\left(1-x^{1 / p}\right) \varsubsetneqq S\left(1-x^{1 / p^{2}}\right) \varsubsetneqq \cdots \varsubsetneqq S\left(1-x^{1 / p^{n}}\right) \varsubsetneqq \cdots,
$$

a strictly ascending chain of principal ideals, hence divisorial ideals. This is a contradiction.

\section{References}

[1] S. A. Amitsur and L. W. Small, 'Prime ideals in PI-rings', J. Algebra 62 (1980), 358-383.

[2] N. Bourbaki, Commutative algebra, Vol. 8 (Hermann, Paris, 1972).

[3] G. Cauchon, Lès T-anneaux et les anneaux à identités polynomiales Noétheriens (Thèse, Université de Paris XI, 1977).

[4] M. Chamaire, Anneaux de Krull non commutatifs (Thèse, Université Claude-Bernard-Lyon I, 1981).

[5] M. Chamarie, 'Anneaux de Krull non commutatifs', J. Algebra 72 (1981), 210-222. 
[6] L. G. Chouinard II, 'Krull semigroups and divisor class groups', Canad. J. Math. 23 (1981), 1459-1468.

[7] R. M. Fossum, 'Maximal orders over Krull domains', J. Algebra 10 (1968), 321-332.

[8] R. M. Fossum, The divisor class group of a Krull domain (Springer Verlag, Berlin, 1973).

[9] R. Gilmer, Multiplicative ideal theory (Marcel Dekker, New York, 1972).

[10] R. Gilmer and T. Parker, 'Divisibility properties in semigroup rings', Michigan Math. J. 21 (1974), 65-86.

[11] M. D. Larsen and P. J. McCarthy, Multiplicative theory of ideals (Academic Press, New York, 1971).

[12] R. Matsuda, 'On algebraic properties of infinite group rings', Bull. Fac. Sci. Ibaraki Univ. Series A 7 (1975), 29-37.

[13] G. Maury and J. Raynaud, Ordres maximaux au sens de K. Asano (Lecture Notes in Math. Vol. 808, Springer Verlag, Berlin, 1980).

[14] D. S. Passman, 'It's essentially Maschke's theorem', Rocky Mountain J. Math. 13 (1983), 37-54.

[15] J. C. Robson and L. W. Small, 'Hereditary prime P.I. rings are classical hereditary orders', $J$. London Math. Soc. 8 (1974), 499-503.

[16] L. H. Rowen, Polynomial identities in ring theory (Academic Press, New York, 1977).

[17] W. Schelter, 'Integral extensions of rings satisfying a polynomial identity', J. Algebra 40 (1976), 245-257.

[18] P. Wauters, 'On $\Omega$-Krull rings in the graded sense', Bull. Soc. Math. Belg. 35 (1983), 1-26.

[19] P. Wauters, 'On some subsemigroups of noncommutative Krull rings', Comm. Algebra 12 (1984), 1751-1765.

Department of Mathematics

Katholieke Universiteit Leuven

University of Cape Town

Leuven

7700 Rondebosch

Belgium

South Africa 